\title{
EXISTENCE OF APPROXIMATE EQUILIBRIA AND CORES
}

\author{
By Werner Hildenbrand, David SchmeIdler, and Shmuel ZamiR ${ }^{1}$
}

IT IS WELL KNOWN that for a finite exchange economy, where preferences are not assumed to be convex, there may be no price equilibrium or even the core may be empty. For this reason it was proposed to enlarge the set of price equilibria and the core by introducing the concepts of "approximate equilibrium" and "approximate core."

The existence of approximate equilibria for exchange economies, where preferences are not assumed to be convex, has been investigated by R. Starr [6]. He showed that there exists a "quasi-equilibrium," provided the number of participants is large enough and there is a bound on the "degree of non-convexity" $[6$, p. 30, Assumption D]. In this note we shall show the existence of "approximate equilibria" (a stronger concept than the one considered by Starr [6, p. 31]) for large economies where the preferences are neither assumed to be convex nor complete. To obtain our result we shall assume that the preferences and the endowments of all participating agents belong to a compact set.

In [5] Shapley and Shubik proved that, for a large replica of a given economy with transferable utility, the $\varepsilon$-core is nonempty. We shall generalize this result to large economies without transferable utility by using the concept of $\varepsilon$-core as introduced by Kannai [2]. The nonemptiness of the $\varepsilon$-core follows easily from the existence of approximate equilibria and a relationship between the set of approximate equilibria and $\varepsilon$-core.

The existence of $\varepsilon$-core for large economies (with a fixed number of types) can also be deduced from Kannai's Theorem $C^{\prime}[2]$ in its stronger form (Theorem $C^{\prime \prime}$ in [3]).

\section{THE MODEL AND STATEMENT OF RESULTS}

Let $T$ denote an infinite set. For every $t$ in $T$, there is defined a preference relation $\succ_{t}$ on the positive orthant $\Omega$ of the $d$-dimensional Euclidian space $R^{d}(d \geqslant 2)$ (i.e., $\succ_{t} \subset \Omega \times \Omega$; we will write $x \succ_{t} y$ instead of $(x, y) \in \succ_{t}$ ). We assume throughout this paper that for every $t$ in $T$ the relation $\succ_{t}$ is irreflexive (for all $x$ in $\Omega$ not $x \succ_{t} x$ ), transitive (for all $x, y$, and $z$ in $\Omega ; x \succ_{t} y$ and $y \succ_{t} z$ imply $x \succ_{t} z$ ), open (the set $\succ_{t}$ is open in the relative topology of $\Omega \times \Omega$ ), and strongly monotonic (for all $x$ and $y$ in $\Omega: x \geqslant y$ and $x \neq y$ imply $x \succ_{t} y$ ). (Inequalities between vectors are

\footnotetext{
${ }^{1}$ The research of W. Hildenbrand and S. Zamir was done in part during Summer 1971 at the University of California, Berkeley, under a grant from the National Science Foundation. The research of D. Schmeidler was done during Summer 1971 at the University of California, Berkeley, and was supported by grant GS-3274 from the National Science Foundation.
} 
assumed to hold relative to coordinates.) We also define, for every $t$ in $T$, a vector $w_{t}$ in $\Omega, w_{t} \neq 0$ (the initial endowment of trader $t$ ).

We denote by $S$ the open simplex

$$
\left\{p=\left(p^{1}, \ldots, p^{d}\right) \in \Omega \mid \sum_{i=1}^{d} p^{i}=1 \text { and } p^{i}>0 \text { for all } i\right\}
$$

in $R^{d}$. The demand correspondence $\psi: T \times S \rightarrow \Omega$ is defined by:

$$
\psi(t, p)=\left\{x \in \Omega \mid p x \leqslant p w_{t} \text { and } y \succ_{t} x \text { implies } p y>p w_{t}\right\} .
$$

It is shown in [4] that $\psi$ is well-defined and, by the monotonicity assumption, $p x=p w_{t}$ holds for all $x$ in $\psi(t, p)$. An exchange economy is by definition a finite subset, say $E$, of $T$. An allocation in the economy $E$ is a collection $\left\{x_{t}\right\}_{t \in E}$ of elements of $\Omega$ satisfying $\Sigma_{t \in E}\left(x_{t}-w_{t}\right)=0$. In what follows we shall make use of two assumptions on the set $T$ of potential traders:

Assumption 1-Boundedness of Initial Endowments: There is a positive real number $M$ such that $\left|w_{t}\right| \leqslant M$ for all $t$ in $T\left(|\cdot|\right.$ being the Euclidean norm in $\left.R^{d}\right)$.

To state our second assumption we need a mathematical concept which reflects the intuitive idea of "similar agents." This concept is a topology on the set of preferences. A precise definition of the topology is postponed to Appendix 1, since it is applied explicitly only in Appendix 2, while through the rest of the paper we need only know that there is such a topology.

Assumption 2-Compactness of Preferences: The set $\left\{\succ_{t}\right\}_{t \in T}$ is compact.

We may note that Assumption 2 is fairly weak since, for instance, the set of all irreflexive, transitive, open, and monotonic ${ }^{2}$ preferences is compact in our topology. ${ }^{3}$

Before we state the main theorem we need a few more notations. For a set $A$ we denote by $|A|$ the number of its element. For $a \in R^{d}$ and $A \subset R^{d}$, let $\rho(a, A)=$ $\inf _{b \in A}|a-b|$. Further, $e$ will denote the vector $(1, \ldots, 1) \in R^{d}$.

THEOREM 1: If T satisfies Assumptions 1 and 2, then for every $\varepsilon>0$ and $\delta>0$, there is an integer $\bar{n}$ such that for every economy $E$ in $T$ with $\Sigma_{t \in E} w_{t}>|E| \delta e$ and $|E|>\bar{n}$ there is an allocation $\left\{x_{t}\right\}_{t \in E}$ and a price vector $p \in S$ such that : (i) for each $t \in E, p x_{t}=p w_{t}$ and $\rho\left(x_{t}, \psi(t, p)\right)<\varepsilon ;$ (ii) $\left|\left\{t \in E \mid x_{t} \notin \psi(t, p)\right\}\right|<\bar{n}$.

An allocation satisfying (i) and (ii) will be referred to as $\bar{n}$-bounded $\varepsilon$-equilibrium. It follows from (i) and (ii) that the aggregate deviation of the traders' bundles from their demands is bounded by a bound independent of the number of traders in the economy, namely $\Sigma_{t \in E} \rho\left(x_{t}, \psi(t, p)\right)<\varepsilon \bar{n}$.

\footnotetext{
${ }^{2}$ A preference relation $>$ is said to be monotonic if $x>y$ implies $x>y$.

${ }^{3}$ For the proof of this fact which will not be given here we are grateful to B. Grodal.
} 
A coalition $C$ is a nonempty subset of $E$. Given $x$ and $y$ in $\Omega$, we denote by $x \ominus y$ the vector whose $j$ th coordinate is $\max \left\{0, x^{j}-y^{j}\right\}$ for $1 \leqslant j \leqslant d$.

Let $\left\{x_{t}\right\}$ be an allocation of an economy $E$, and let $\varepsilon$ be a positive number. The allocation $\left\{x_{t}\right\}$ is said to be $\varepsilon$-blocked if there is a coalition $C \subset E$ and an allocation $\left\{y_{t}\right\}$ such that: (i) $y_{t} \succ_{t} x_{t}$ for every $t \in C$; (ii) $\Sigma_{t \in C} y_{t} \leqslant\left(\Sigma_{t \in C} w_{t}\right) \ominus|C| \varepsilon e$. The set of all allocations which are not $\varepsilon$-blocked is called the $\varepsilon$-core of $E .^{4}$

For the next result we add the assumption that each trader has a complete preference relation on $\Omega$. In our notation this means actually that for all $t$ in $T$ and all $x, y$, and $z$ in $\Omega: x \nsucc_{t} y$ and $y \nsucc_{t} z$ implies $x \nsucc_{t} z$. (Here $x \ngtr_{t} y$ means: not $x \succ_{t} y$.) When adding this assumption we have the following theorem.

THEOREM 2: For every $\varepsilon>0$ and $\delta>0$ there is an integer $\bar{n}$ such that every economy $E$ in $T$ with $\Sigma_{t \in E} w_{t}>|E| \delta e$ and $|E|>\bar{n}$ has a nonempty $\varepsilon$-core.

\section{PROOFS}

In the proof of Theorem 1 we use the following two lemmas.

LEMMA 1 (Shapley-Folkman): Let $\left\{Q_{i}\right\}_{i=1}^{n}$ be a collection of nonempty subsets of $R^{d}$. Let $x_{i}$ be in the convex hull of $Q_{i}$ for $i=1,2, \ldots, n$. Then for each i there is $y_{i}$ in the convex hull of $Q_{i}$ such that $\sum_{i=1}^{n}\left(x_{i}-y_{i}\right)=0$ and with the possible exception of at most $d$ indices $y_{i}$ is in $Q_{i}$.

This lemma was used by Starr, and its proof appears in his paper [6, Appendix 2, Lemma 2 and Corollary].

LEMMA 2: Under the conditions of the theorem, there is a number $\eta>0$ such that for every economy $E$ with $\Sigma_{t \in E} w_{t} \geqslant|E| \delta$ and every competitive price $p \in S$ of the continuous representation of $E$ it follows that $p \geqslant \eta e$.

The proof of this lemma is postponed to Appendix 2, but let us explain here the concept of a "continuous representation of a finite economy" (as used by Kannai [2]). Given $E$, we define the following economy with a continuum of traders (Aumann's model). Let $\left\{I_{t}\right\}_{t \in E}$ be a partition of the unit interval such that for all $t$, $I_{t}$ is Lebesgue measurable of measure $1 /|E|$. For every $s$ in the unit interval we define the initial endowment of $s$ to be $w_{t}$ and its preference to be $\succ_{t}$ if $s$ is in $I_{t}$. It is obvious that the economy defined in this way fulfills the conditions for existence of competitive equilibrium with incomplete preferences [4].

Proof of Theorem 1 : Let $(p, f(\cdot))$ be a competitive equilibrium in the continuous representation of an economy $E$. For $t$ in $E$ we define $x_{t}=|E| \int_{I t} f(s) d s$. Then $x_{t}$ is in conv $(\psi(t, p))$. By Lemma 1 there are $y_{t}$ in $\Omega$ for $t$ in $E$ such that: (a) $\Sigma_{t \in E}\left(x_{t}-y_{t}\right)=0$, i.e., $\left\{y_{t}\right\}_{t \in E}$ is an allocation in $E$, and (b) $y_{t}$ is in $\psi(t, p)$ with the possible exception of at most $d$ traders $t$ in $E$, and even then $y_{t}$ is in the convex hull of $\psi(t, p)$. In particular this implies that $p y_{t}=p w_{t}$ for all $t$ in $E$. Using the inequality

\footnotetext{
${ }^{4}$ This concept has been introduced by Kannai [2] under the name of "weak-E-core."
} 
$p \geqslant \eta e$ obtained from Lemma 2 and Assumption $1\left(\left|w_{t}\right| \leqslant M\right.$ for all $\left.t\right)$, we conclude that $\left|y_{t}-x\right| \leqslant d M / \eta$ for all $x$ in $\Omega$ such that $p x=p w_{t}$. Next, we define $z_{t}$ as follows: $z_{t}=y_{t}$ if $y_{t} \in \psi(t, p)$ and $z_{t}$ is an arbitrary point in $\psi(t, p)$ if $y_{t} \notin \psi(t, p)$. Let $z=$ $\Sigma_{t \in E}\left(w_{t}-z_{t}\right)$; then $z=\Sigma_{t \in E}\left(y_{t}-z_{t}\right)$. Hence $|z| \leqslant d^{2} M / \eta$. Clearly $p z=0$. The crucial step in our proof is to change the vectors $z_{t}$ slightly so that the change of each $z_{t}$ is less than $\varepsilon$ and the new vectors form an allocation. To do this we construct $d$ disjoint subsets $\left\{E_{i}\right\}_{i=1}^{d}$ of $E$ such that

$$
\begin{aligned}
& z_{t}^{i}>\delta / d \quad \text { for all } \quad t \in E_{i}, \\
& \min \{\varepsilon, \delta / d\} \cdot\left|E_{i}\right|>\left|z^{i}\right| .
\end{aligned}
$$

We show later that there is an integer $\bar{n}$ such that this construction is possible provided $|E|>\bar{n}$, and in that case $\left|\cup_{i} E_{i}\right|<\bar{n}$. The change of $z_{t}$ is done now as follows: denote

$$
D^{+}=\left\{j \mid 1 \leqslant j \leqslant d ; z^{j} \geqslant 0\right\}
$$

and

$$
D^{-}=\left\{j \mid 1 \leqslant j \leqslant d ; z^{j}<0\right\} .
$$

If $D^{-}=\varnothing$, then $z=0$ (since $p z=0$ ) and $\left\{z_{t}\right\}$ is already an allocation. Suppose, therefore, that $i \in D^{-}$; consequently $D^{+} \neq \varnothing$. Let $\left\{a^{j}\right\}_{j \in D^{+}}$be any positive numbers such that $\Sigma_{j \in D^{+}} p^{j} a^{j}=p^{i}\left|z^{i}\right|$ and define $a^{i}=z^{i}$ and $a^{j}=0$ for $j \in D^{-} \backslash\{i\}$. We define now $\bar{z}_{t}=z_{t}+a /\left|E_{i}\right|$ for $t \in E_{i}$ and $\bar{z}_{t}=z_{t}$ for $t \in E \backslash E_{i}$. By construction of $a$ and by the definition of $E_{i}, \bar{z}_{t} \in \Omega, a /\left|E_{i}\right|<\varepsilon, p a=0$, and hence $p \bar{z}_{t}=p w_{t}$ for all $t \in E$. Furthermore, for $t \in E_{i}$ we have $\rho\left(\bar{z}_{t}, \psi(t, p)\right)<\varepsilon$ while for $t \in E \backslash E_{i}, \bar{z}_{t} \in \psi(t, p)$. If we denote $\bar{z}=\Sigma_{t \in E}\left(w_{t}-\bar{z}_{t}\right)$, then $\left\{j \mid 1 \leqslant j \leqslant d ; \bar{z}^{j}<0\right\}=\left|D^{-}\right|-1$. Repeating this procedure a finite number of times (e.g., $\left|D^{-}\right|$) we end up with an allocation as required in the theorem.

Left to be shown is the existence of $\bar{n}$ for which the above mentioned $\left\{E_{i}\right\}_{i=1}^{d}$ can be constructed. For any $i(1 \leqslant i \leqslant d)$, we define $\overline{\bar{E}}_{i}=\left\{t \in E \mid y_{i}^{t}>\delta / d\right\}$; then

$$
|E| \delta<\sum_{t \in E} y_{t}^{i} \leqslant\left|\overline{\bar{E}}_{i}\right| d M / \eta+\left(|E|-\left|\overline{\bar{E}}_{i}\right|\right) \delta / d .
$$

The first inequality follows from the condition $\Sigma_{t \in E} w_{t}>|E| \delta e$. The second follows from the definition of $\overline{\bar{E}}_{i}$ and from the bound on $y_{t}$ obtained previously. This implies $\left|\overline{\bar{E}}_{i}\right| \geqslant|E| \delta \eta /(2 d M)$. Let

$$
N=\max \left\{d^{2} M /(\eta \varepsilon), d^{3} M /(\eta \delta)\right\}
$$

and let $\bar{n}$ be any integer greater than $2 d^{2}(N+1) M /(\delta \eta)$. It is easily verified that $|E|>\bar{n}$ implies $\left|\overline{\bar{E}}_{i}\right|>d N+d$. Now we define $\widetilde{E}_{i}$ to be $\overline{\bar{E}}_{i}$ if $\left|\overline{\bar{E}}_{i}\right| \leqslant \bar{n}$ and to be any subset of $\overline{\bar{E}}_{i}$ with cardinality $\bar{n}$ otherwise. Since $(2 d / \eta)(M / \delta) \geqslant 1$, we obtain $d N+$ $d \leqslant \widetilde{E}_{i} \leqslant \bar{n}$.

Let

$$
\bar{E}_{i}=\left\{t \in \widetilde{E}_{i} \mid z_{t}^{i}>\delta / d\right\}
$$

Since $z_{t} \neq y_{t}$ for at most $d$ traders we have $d N \leqslant\left|\bar{E}_{i}\right| \leqslant \bar{n}$. Having the sets $\bar{E}_{1}, \ldots, \bar{E}_{d}$, 
our last step is to choose for each $i$ a subset $E_{i}$ of $\bar{E}_{i}$ such that $E_{1}, \ldots, E_{d}$ are disjoint, $\left|E_{i}\right| \geqslant N$ and $\left|U_{i} E_{i}\right| \leqslant \bar{n}$. Such a choice is obviously possible. Finally, condition (a) trivially holds while condition (b) follows from $\left|E_{i}\right| \geqslant N$, the definition of $N$, and the inequality $|z|<d^{2} M / \eta$.

Q.E.D.

To prove Theorem 2, let us prove first the following proposition.

Proposition: For every $\varepsilon>0$ there is an $\varepsilon^{\prime}>0$ such that if $\left(\left\{x_{t}\right\}, p\right)$ is an $\varepsilon^{\prime}-$ equilibrium of an economy $E$ where $p \geqslant \eta$, then for every $t \in E$ :

$$
y_{t} \succ_{t} x_{t} \Rightarrow p y_{t}>p w_{t}-\varepsilon \eta \text {. }
$$

PROOF: Assume that the proposition is false. So there is a sequence of (without a loss of generality, disjoint) economies $\left\{E_{n}\right\}_{n=1}^{\infty}$ and for each $E_{n}$ there is a $(1 / n)$ equilibrium $\left(\left\{x_{t}\right\}_{t \in E_{n}}, p_{n}\right)$, a $t_{n} \in E_{n}$, and $y_{n} \in \Omega$ such that

$$
y_{n} \succ_{t_{n}} x_{t_{n}} \quad \text { and } \quad p_{n} y_{n} \leqslant p_{n} w_{t_{n}}-\varepsilon \eta \text {. }
$$

By compactness and completeness assumptions there are converging subsequences of $\left\{y_{n}\right\},\left\{x_{t_{n}}\right\},\left\{p_{n}\right\},\left\{\succ_{t_{n}}\right\}$, and $\left\{w_{t_{n}}\right\}$ with the limit points $y_{0}, x_{0}, p_{0}, \succ_{t_{0}}$, and $w_{0}$ respectively satisfying: (i) $x_{0} \nsucc_{t_{0}} y_{0}$ and (ii) $p_{0} y_{0} \leqslant p_{0} w_{0}-\varepsilon \eta$. By the definition of $(1 / n)$-equilibria, $\rho\left(x_{t_{n}}, \psi\left(t_{n}, p_{n}\right)\right)<(1 / n)$. Now $p_{n} \geqslant \eta e$ implies that $\psi\left(t_{n}, p_{n}\right)$ are uniformly bounded. Since $\psi$ is upper hemicontinuous [1, Appendix A, IV], it follows that $x_{0} \in \psi\left(t_{0}, p_{0}\right)$ in contradiction to (i) and (ii) and monotonicity of $\succ_{t_{0}}$.

Proof of Theorem 2: Given $\varepsilon>0$ and $\delta>0$, there is an $\eta>0$ determined by Lemma 2 . Let $\varepsilon^{\prime}>0$ be determined by the proposition.

According to Theorem 1 there is an integer $\bar{n}$ such that for every economy $E$ with $|E|>\bar{n}$ and $\sum_{t \in E} w_{t}>|E| \delta e$ there is an $\varepsilon^{\prime}$-equilibrium $\left(\left\{x_{t}\right\}, p\right)$. Furthermore, we know from the proof of Theorem 1 that there is an $\varepsilon^{\prime}$-equilibrium with $p$ satisfying $p \geqslant \eta e$. We claim that such an $\varepsilon^{\prime}$-equilibrium of $E$ is in the $\varepsilon$-core of $E$.

Assume to the contrary that the allocation $\left\{x_{t}\right\}$ is $\varepsilon$-blocked, i.e., there is a coalition $C$ and an allocation $\left\{y_{t}\right\}$ such that

$$
y_{t} \succ_{t} x_{t} \text { for every } t \in C,
$$

and

$$
\sum_{t \in C} y_{t} \leqslant\left(\sum_{t \in C} w_{t}\right) \ominus|C| \varepsilon e
$$

By the proposition, $p y_{t}>p x_{t}-\varepsilon \eta$ for all $t \in C$. Hence,

$$
p \sum_{t \in C} y_{t}>p \sum_{t \in C} w_{t}-|C| \varepsilon \eta
$$


Let

$$
\left(\sum_{t \in C} w_{t}\right) \ominus|C| \varepsilon e=\sum_{t \in C} w_{t}-\left(\alpha^{1}, \ldots, \alpha^{d}\right)
$$

Without loss of generality we can assume that the $\varepsilon$-blocking coalition $C$ is such that for every $t \in C$ there is a coordinate $j_{t}$ such that $w_{t}^{j_{t}} \geqslant \varepsilon$ (otherwise, $C^{t} \backslash\{t\}$, $\varepsilon$-blocks $\left\{x_{t}\right\}$ via the same allocation $\left.\left\{y_{t}\right\}\right)$. Hence, $\Sigma_{i=1}^{d} \alpha^{j} \geqslant|C| \varepsilon$ and consequently

$$
p \sum_{t \in C} y_{t} \leqslant p\left[\left(\sum_{t \in C} w_{t}\right)-|C| \varepsilon e\right] \leqslant p \sum_{t \in C} w_{t}-|C| \varepsilon \eta,
$$

in contradiction to (3).

Bonn University and Université Catholique de Louvain,

Tel-Aviv University,

and

Hebrew University of Jerusalem.

Manuscript received November, 1971 ; revision received April, 1972.

\section{APPENDIX 1}

Topology on the Preferences $\left\{\succ_{t}\right\}_{t \in T}$

For every $t$ in $T$ let

$$
F_{t}=\left\{(x, y) \in \Omega \times \Omega \mid x \nsucc_{t} y\right\} .
$$

The sets $F_{t}$ are closed in $R^{2 d}$ and so we can use the following well-known topology on closed subsets of Euclidian space: the sub-base of the topology consists of the sets

$$
\left\{F_{t} \mid F_{t} \cap K=\varnothing \text { and } F_{t} \cap U \neq \varnothing\right\}
$$

where $K$ is a compact set and $U$ is an open set in $R^{2 d}$.

A sequence $\left(\succ_{t_{n}}\right)_{n=1, \ldots}$ of preferences converges to a preference $\succ_{t}$ for this topology if and only if

$$
\operatorname{Lim} \operatorname{Inf} F_{t_{n}}=F_{t}=\operatorname{Lim} \operatorname{Sup} F_{t_{n}},
$$

where $\operatorname{Lim} \operatorname{Inf} F_{t_{n}}$ is the set of points $(x, y) \in \Omega \times \Omega$ such that every neighborhood of $(x, y)$ intersects all the $F_{t_{n}}$ with sufficiently large $n$ and $\operatorname{Lim} \operatorname{Sup} F_{t_{n}}$ is the set of points $(x, y) \in \Omega \times \Omega$ such that every neighborhood of $(x, y)$ intersects infinitely many $F_{t_{n}}$. For a more detailed discussion of this topology and its equivalent formulations, see [1, pp. 164-165]. (References to mathematical works on this topology also appear in $[\mathbf{1}]$.)

\section{APPENDIX 2}

\section{Proof of Lemma}

Suppose that the lemma is false and there is a sequence of economies $\left\{E_{n}\right\}_{n=1}^{\infty}$ and a sequence of competitive prices (in $S$ ) $\left\{p_{n}\right\}_{n=1}^{\infty}$ in their corresponding continuous representations such that, without loss of generality, $p_{n} \rightarrow p$ and $p^{1}=0$. In order to simplify notations, we assume without loss of generality that the economies in $\left\{E_{n}\right\}$ are disjoint. We use the notations of the first paragraph in the proof of the 
theorem. Thus, for all $n,\left\{x_{t}\right\}_{t \in E_{n}}$ is an allocation in $E_{n}$ and $x_{t}$ is in the convex hull of $\psi(t, p)$ for all $t$ in $E_{n}$. Define $\bar{E}_{n}=\left\{t \in E_{n}|| x_{t} \mid<k M\right\}$ for some fixed positive number $k$. Then, by Assumption 1,

$$
\left|E_{n}\right| M \geqslant \sum_{t \in E_{n}}\left|w_{t}\right| \geqslant\left|\sum_{t \in E_{n}} w_{t}\right|=\left|\sum_{t \in E_{n}} x_{t}\right| \geqslant\left|\sum_{t \in E_{n}}\right| x_{t}|| / d \geqslant\left(\left|E_{n}\right|-\left|\bar{E}_{n}\right|\right) k M / d .
$$

Hence, $\left|\bar{E}_{n}\right| /\left|E_{n}\right| \geqslant(k-d) / k$.

Next let $D=\left\{i \mid 1 \leqslant i \leqslant d, p^{i}>0\right\}$. Then there is $\alpha>0$ such that $p_{n}^{i}>\alpha$ for all $i$ in $D$ and all $n$. Hence, for every $i$ in $D$ and every $t$ in $E_{n}$, we have

$$
\alpha x_{t}^{i} \leqslant p_{n} x_{t} \leqslant p_{n} w_{t} \leqslant M .
$$

The last inequality holds by Assumption 1 on $T$. So $x_{t}^{i} \leqslant M / \alpha$. Let

$$
E_{n}^{\imath}=\left\{t \in E_{n} \mid x_{t}^{\imath}>\delta / k\right\}
$$

then

$$
\left|E_{n}\right| \delta \leqslant \sum_{t \in E_{n}} w_{t}^{l}=\sum_{t \in E_{n}} x_{t}^{l} \leqslant\left(\left|E_{n}\right|-\left|E_{n}^{l}\right|\right) \delta / k+\left|E_{n}^{l}\right| M / \alpha
$$

where $i$ is in $D$ and $\delta$ is from the statement of Lemma 2 (and the theorem). Finally we obtain the inequality

$$
\left|E_{n}^{\prime}\right| /\left|E_{n}\right| \geqslant(\delta \alpha / M)(k-1) / k .
$$

Now, for any positive numbers $\delta, \alpha, M$, and $d$ there is $k$ such that

$$
(\delta \alpha / M)(k-1) / k+(k-d) / k>1,
$$

i.e., for this $k, \bar{E}_{n} \cap E_{n}^{i} \neq \varnothing$ for all $n$. We denote by $t_{n}$ an element of $\bar{E}_{n} \cap E_{n}^{i}$ for $n=1,2, \ldots$, .

Let $q$ be a vector in $\Omega$ such that $q^{i}=0$ for $i$ in $D$ and $q^{i}(d-|D|)=1$ for $i \notin D$. Since $x_{t_{n}}$ is in $\operatorname{conv} \psi\left(t_{n}, p_{n}\right)$, the intersection

$$
\psi\left(t_{n}, p_{n}\right) \cap\left\{x \in \Omega \mid q x \leqslant q x_{t_{n}}\right\}
$$

is nonempty. Denote by $y_{n}$ an element of this intersection.

Every $y_{n}, n=1,2, \ldots$, satisfies the inequalities

$$
p_{n}^{l} y_{n}^{l} \leqslant p_{n} y_{n}=p_{n} w_{t_{n}} \leqslant M
$$

and

$$
q^{l} y_{n}^{i} \leqslant q y_{n} \leqslant q x_{t_{n}} \leqslant k M
$$

for $i=1, \ldots, d$. Hence, $y_{n}^{l} \leqslant M / \alpha$ for $i$ in $D$ and $y_{n}^{l} \leqslant d k M$ for $i \notin D$. Consequently $\left|y_{n}\right| \leqslant d^{2} k M / \alpha$ for all $n$. So the compactness of $\left\{\succ_{t_{n}}\right\}_{n=1}^{\infty}$ and of $\left\{y_{n}\right\}_{n=1}^{\infty}$ imply that there is a subsequence (and to simplify notations, assume that this is the original sequence), a preference relation $\succ_{t_{0}}$, and $y_{0}$ in $\Omega$ such that $>_{t_{n}} \rightarrow>_{t_{0}}$ and $y_{n} \rightarrow y_{0}$.

Since $p_{n} y_{n}=p_{n} x_{n} \geqslant p_{n}^{i} x_{n}^{i}>\alpha \delta / k$, for every $n$ there is a coordinate $j(n)$ such that $y_{n}^{j(n)}>\alpha \delta /(d k)$. As $p_{n}^{\prime} \rightarrow 0$ for $j \notin D$, it follows that for $n$ large enough $j(n) \in D$. By choosing a subsequence of $\left\{y_{n}\right\}$ (and to simplify notations we denote it also by $\left.\left\{y_{n}\right\}\right)$, there is a coordinate $j$ in $D$ such that $y_{n}^{j}>\alpha \delta /(d k)>0$ for all $n$.

We denote by $e_{j}$ the unit vector in $R^{d}$ whose $j$ th coordinate is 1 . For each $n$ let $\theta_{n}$ be defined by

$$
p_{n}\left(y_{n}+q-\theta_{n} y_{n}^{j} e_{j}\right)=p_{n} y_{n} .
$$

Clearly $\theta_{n} \rightarrow 0$. Hence, for $n$ large enough, the vectors $z_{n}$ defined by

$$
z_{n}=y_{n}+q-\theta_{n} y_{n}^{j} e_{j}
$$

are in $\Omega, p_{n} z_{n}=p_{n} y_{n}=p_{n} w_{t_{n}}$, and $z_{n} \rightarrow y_{0}+q$. Since $y_{n}$ is in $\psi\left(t_{n}, p_{n}\right), z_{n} \nsucc_{t_{n}} y_{n}$. In the limit we obtain $y_{0}+q \nsucc_{t_{0}} y_{0}\left[\mathbf{1}\right.$, Appendix A, I], a contradiction to monotonicity of $\succ_{t_{0}}$.

Q.E.D. 


\section{REFERENCES}

[1] Hildenbrand, W.: "On Economies with Many Agents," Journal of Economic Theory, 2 (1970), 161-188.

[2] KannaI, Y.: "Continuity Properties of the Core of a Market," Econometrica, 38 (1970), 791-815.

[3] —: "A Correction," Econometrica, 40 (1972), 955-958.

[4] SCHMeIder, D.: "Competitive Equilibria in Markets with a Continuum of Traders and Incomplete Preferences," Econometrica, 37 (1969), 578-585.

[5] Shapley, L. S., And Shubik, M.: "Quasi-Cores in a Monetary Economy with Nonconvex Preferences," Econometrica, 34 (1966), 805-827.

[6] Starr, R. M.: "Quasi-Equilibria in Markets with Nonconvex Preferences," Econometrica, 37 (1969), 25-38. 